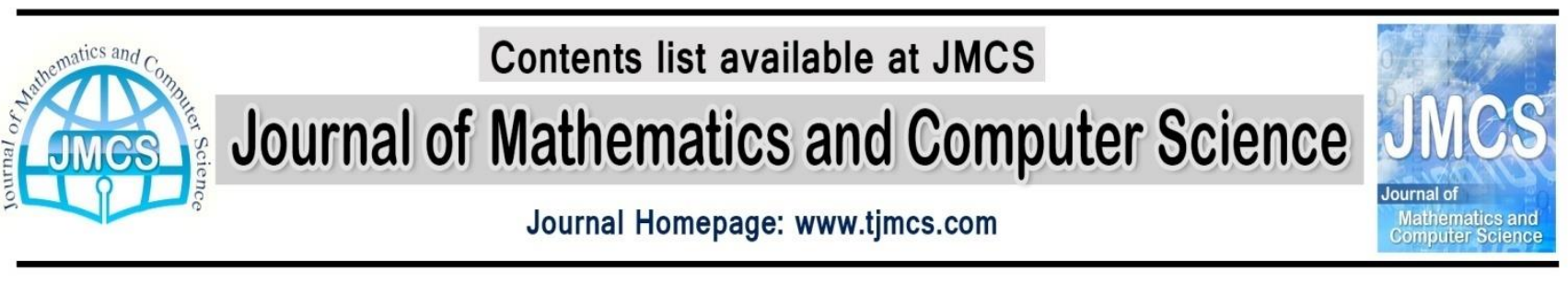

\title{
Some Algebraic Structures of Languages
}

\author{
Dasharath Singh ${ }^{1, *}$, Ahmed Ibrahim Isah ${ }^{1,+}$ \\ ${ }^{1}$ Mathematics Department, Ahmadu Bello University, Zaria, Nigeria. \\ *mathdss@yahoo.com \\ +aisah204@gmail.com
}

Article history:

Received November, 2014

Accepted January, 2015

Available online January 2015

\begin{abstract}
In this paper, suitable operations are defined on the class of partitions of a language which give rise to certain monoids and semigroups. In particular, certain algebraic structures of a language defined over a string are described.
\end{abstract}

Keywords: Language, partition, semigroup, monoid

Mathematics Subject Classification: 11P83, 68Q45, 68Q70

\section{Introduction}

The applications of various algebraic structures abound (see [1, 2, 3, 6, 7, 8] for details and related references). In particular, certain algebraic structures have found applications in formal language theory (see [6] for details). Moreover, a number of algebraic structures of partitions of a set and that of an integer have been developed which have useful applications in computer arithmetic, formal languages and sequential machines (see [5, 7] for details). In this paper, suitable operations on the set of partitions of a language are defined which give rise to certain monoids and semigroups. In addition, certain algebraic structures of a language defined over a string are described. 


\section{Definitions}

\section{Definition 2.1 Union, Intersection and Concatenation of Languages}

Let $X$ be an alphabet and $X^{*}$ denote the set of all strings over $X$. A language $L$ is a subset of $X^{*}$ i.e., $L \subseteq X^{*}$. Let $L_{1}$ and $L_{2}$ be any two languages over $X$. The union of $L_{1}$ and $L_{2}$, denoted $L_{1} \cup L_{2}$, is the language $L_{1} \cup L_{2}=\left\{u \in X^{*} \mid u \in L_{1}\right.$ or $\left.u \in L_{2}\right\}$. The intersection of $L_{1}$ and $L_{2}$, denoted $L_{1} \cap L_{2}$, is the language $L_{1} \cap L_{2}=\left\{u \in X^{*} \mid u \in L_{1}\right.$ and $\left.u \in L_{2}\right\}$. The concatenation (or simply, catenation) of $L_{1}$ and $L_{2}$, denoted $L_{1} L_{2}$, is the language $L_{1} L_{2}=$ $\left\{u=u_{1} u_{2} \mid u_{1} \in L_{1}\right.$ and $\left.u_{2} \in L_{2}\right\}$. It is immediate to see that the union, intersection and catenation of languages are each associative because union, intersection and catenation of strings are each associative and hence $X^{*}$ with catenation is a non-commutative monoid (see $[2,4]$, for details). In the same vein, let $u^{*}$ be defined as the set of all strings over $u \in X^{*}$, then $u^{*}$ with catenation is a commutative monoid.

\section{Definition 2.2 Cardinality bounded languages}

Let $X^{n *}$, henceforth called a cardinality bounded language over $X$, denote the set of all strings of length $\leq n$ over $X$. In other words, $\left\{X^{n *}\right\}$ is a strictly monotonic increasing nested sequence, and obviously $X^{*}=X^{0 *} \cup X^{1 *} \cup \ldots \cup X^{n *} \cup \ldots$. However, it gives an alternative form of representation of the usual one viz., $X^{*}=X^{0} \cup X^{1} \cup \ldots \cup X^{n} \cup \ldots$..., where $X^{n}$ is the set of all strings of length $n$ over $X$. Moreover, $\cup_{n=0}^{\infty} X^{n}=\bigcup_{n=0}^{\infty} X^{n *}$, but $\bigcap_{n=0}^{\infty} X^{n}=\emptyset$, whereas $\bigcap_{n=0}^{\infty} X^{n *}=\{\varepsilon\}$.

It may also be observed that each of $X^{n *}$ is a well-ordered set with $\subset$ (inclusion), and hence a finite ordinal, say $\alpha, \beta, \gamma, \ldots$, satisfying the following properties: (i) $\beta \in \alpha \Rightarrow \beta \subset \alpha$ (ii) each $\alpha$ is well-ordered by $\subset$ and (iii) neither $\alpha$ nor its element is an element of itself.

For example, let $X=\{0,1\}$, then $X^{0 *}=\{\varepsilon\}, X^{1 *}=\{\varepsilon, 0,1\}, X^{2 *}=\{\varepsilon, 0,1,00,01,10,11\}$, $X^{3 *}=\{\varepsilon, 0,1,00,01,10,11,000,001,010,011,100,101,110,111\}$, and so on.

Let us recapitulate that the cardinality of a language $L$, denoted $|L|$, is the number of strings in $L$. Thus $X^{*}$ is countably infinite over any $X$. Moreover, $\left|X^{n *}\right|=\left|X^{0}\right|+\left|X^{1}\right|+\left|X^{2}\right|+$ $\cdots+\left|X^{n}\right|$.

\section{Examples}

Let $X=\{0\}$. Then,

$$
\begin{gathered}
\left|X^{n *}\right|=\left|X^{0}\right|+\left|X^{1}\right|+\left|X^{2}\right|+\cdots+\left|X^{n}\right|=|\{\varepsilon\}|+|\{0\}|+|\{00\}|+|\{000\}|+\cdots+\left|\{0\}^{n}\right| \\
=1^{0}+1^{1}+1^{2}+\cdots+1^{n} .
\end{gathered}
$$


Let $X=\{0,1\}$. Then,

$\left|X^{n *}\right|=\left|X^{0}\right|+\left|X^{1}\right|+\left|X^{2}\right|+\cdots+\left|X^{n}\right|=|\{\varepsilon\}|+|\{0,1\}|+|\{00,01,10,11\}|+$

$+|\{000,001,010,011,100,101,110,111\}|+\cdots+\left|\{0,1\}^{n}\right|=2^{0}+2^{1}+2^{2}+\cdots+2^{n}$.

Let $X=\{0,1,2\}$. Then,

$\left|X^{n *}\right|=\left|X^{0}\right|+\left|X^{1}\right|+\left|X^{2}\right|+\cdots+\left|X^{n}\right|=|\{\varepsilon\}|+|\{0,1,2\}|+$

$|\{00,01,02,10,11,12,20,21,22\}|+$

$\mid\{000,001,002,010,011,012,020,021,022,100,101,102,110,111,112,120,121,122,200,201$

$202,210,211,212,220,221,222\}|+\cdots+|\{0,1,2\}^{n} \mid=3^{0}+3^{1}+3^{2}+\cdots+3^{n}$.

Let $X=\{0,1,2,3\}$. Then,

$\left|X^{n *}\right|=\left|X^{0}\right|+\left|X^{1}\right|+\left|X^{2}\right|+\cdots+\left|X^{n}\right|=|\{\varepsilon\}|+|\{0,1,2,3\}|+\mid\{00,01,02,03,10,11,12,13$,

$20,21,21,22,23,30,31,32,33\}|+|\{000,001,002,003,010,011,012,013,020,021,022,023$,

030,031,032,033,100,101,102,103,110,111,112,113,120,121,122,123,130,131,132,133,

$200,201,202,203,210,211,212,213,220,221,222,223,230,231,232,233,300,301,302,303$,

$310,311,312,313,320,321,322,323,330,331,332,333\} \mid=4^{0}+4^{1}+4^{2}+\cdots+4^{n}$.

By induction, if $X$ be a $k$-element set, we have

$\left|X^{n *}\right|=k^{0}+k^{1}+k^{2}+\cdots+k^{n}$.

\section{Some algebraic structures of languages}

\subsection{Monoids of equivalence classes of a partition of a language}

Let $R_{a}$ be a relation on $\mathrm{X}^{*}$ such that for $s, t \in \mathrm{X}^{*}, s R_{a} t$ if and only if $s$ and $t$ are of equal length.

It is easy to see that $R_{a}$ is an equivalence relation on $\mathrm{X}^{*}$ and hence, it partitions $\mathrm{X}^{*}$ into its equivalence classes. In other words, a partition of $X^{*}$ can be viewed as a collection of disjoint languages of $\mathrm{X}^{*}$, whose union is $\mathrm{X}^{*}$.

Let the equivalence class generated by $S \in \mathrm{X}^{*}$ be denoted $[S]_{R_{a}}$ or simply $[S]$, and the quotient set $\mathrm{X}^{*} / R_{a}$ denote the family of all equivalence classes of $\mathrm{X}^{*}$.

Let us define an operation $*$ on $\mathrm{X}^{*} / R_{a}$ such that $[s] *[t]=[s t]$ where $s t$ is the catenation of $s$ and $t$. Then, $\left(\mathrm{X}^{*} / R_{a}, *,[\varepsilon]\right)$ is a monoid of the partition of $X^{*}$ induced by $R_{a}$, where $[\varepsilon]$ 
is the identity of catenation. The operation $*$ is neither commutative nor idempotent, in general. However, the identity element $[\varepsilon]$ is the only idempotent element. Also, the operation * is commutative if $\mathrm{X}$ is a singleton.

Moreover, as described in section 2 above, it is easy to see that $\left(L / R_{a}, *,[\varepsilon]\right)$ is a commutative monoid where $L=\mathrm{u}^{*}, \mathrm{u} \in \mathrm{X}^{*}$.

Similarly, for each of the relations $R_{b}, R_{c}$, and $R_{d}$ defined on $\mathrm{X}^{*}$ as $s R_{b} t$ iff both $s$ and $t$ have the same number of occurrences of each symbol,

(iii) $\quad s R_{d} t$ iff $s$ and $t$ agree in their last symbols;

the respective quotient set is a non-commutative and non-idempotent monoid.

Moreover, each of $R_{b}, R_{c}$, and $R_{d}$, similar to $R_{a}$, defined on a language $u^{*}$, partitions it, and the respective quotient set is a commutative monoid of the partitions of $u^{*}$.

\subsection{Monoids of partitions of a language}

We introduce further three operations on the class of all partitions of $\mathrm{X}^{*}$.

Let $\mathcal{F}\left(\mathrm{X}^{*}\right)$ denote the collection of all partitions of $\mathrm{X}^{*}$ and $S=\left\{S_{1}, S_{2}, \ldots\right\}$ and $T=\left\{T_{1}, T_{2}, \ldots\right\}$ be two partitions of $\mathrm{X}^{*}$. Observe that $S_{i}{ }^{\prime} S$ and $T_{i}{ }^{\prime} s$ are the blocks of $S$ and $T$, respectively, and each block is a subset of $\mathrm{X}^{*}$.

Let a binary operation $\circledast$ be defined on $\mathcal{F}\left(\mathrm{X}^{*}\right)$ as follows:

For any $S, T \in \mathcal{F}\left(\mathrm{X}^{*}\right), S \circledast T$ consists of the set of nonempty intersections of every block of $S$ with every block of $T$. It is clear that the operation $\circledast$ is both associative and commutative as intersection on languages is associative and commutative. The partition consisting of a unique single block is the identity of $\circledast$. It may be observed that $P \circledast P=P$ for all $P \in$ $\mathcal{F}\left(\mathrm{X}^{*}\right)$ i.e., $\circledast$ is idempotent. Thus, $\left(\mathcal{F}\left(\mathrm{X}^{*}\right), \circledast\right)$ or $\left(\mathcal{F}\left(\mathrm{X}^{*}\right), \circledast,\left\{\overline{\mathrm{X}^{*}}\right\}\right)$ is a commutative, idempotent monoid.

Let another binary operation $\bigoplus$ on $\mathcal{F}\left(\mathrm{X}^{*}\right)$ be defined as follows:

Let $S, T \in \mathcal{F}\left(\mathrm{X}^{*}\right)$. A subset $P$ of $\mathrm{X}^{*}$ belongs to $S \oplus T$ if

(i) $\quad P$ is the union of one or more elements of $S$;

(ii) $\quad P$ is the union of one or more elements of $T$; and

(iii) No element of $P$ satisfies (i) and (ii) except $P$ itself.

Clearly, $\bigoplus$ is associative and commutative, and the partition consisting of singleton blocks is the identity of the operation $\bigoplus$ on $\mathcal{F}\left(X^{*}\right)$. Thus, $\left(\mathcal{F}\left(X^{*}\right), \oplus\right)$ or $\left(\mathcal{F}\left(X^{*}\right), \oplus\right.$, $\left.\left\{\overline{\mathrm{x}_{0}}, \overline{\mathrm{x}_{1}}, \overline{\mathrm{x}_{2}}, \ldots\right\}\right)$, where $\mathrm{x}_{i}{ }^{\prime} s$ are the elements of $\mathrm{X}^{*}$, is a commutative, idempotent monoid.

Finally, let a binary operation $\odot$ be defined on $\mathcal{F}\left(\mathrm{X}^{*}\right)$ as follows: 
For any $S, T \in \mathcal{F}\left(\mathrm{X}^{*}\right), S \odot T$ is the union of every block of $S$ with every block of $T$ if no element of the block of $S$ and/or $T$ appears previously. In the case, a block has an element that appeared previously, it is not included in the union.

It is immediate to see that $\odot$ is associative but non-commutative and every element $P \in$ $\mathcal{F}\left(\mathrm{X}^{*}\right)$ is idempotent as $P \odot P=P$ holds. Thus, $\left(\mathcal{F}\left(\mathrm{X}^{*}\right), \bigcirc\right)$ is only a semigroup as there is no identity element.

It is immediate to see that all the foregoing constructions, described above, hold good for $X^{n *}$ as well.

\section{Examples}

Let $X=\{0,1\}$ be an alphabet and $n=2$. Then, $\mathrm{X}^{2 *}=\{\varepsilon, 0,1,00,01,10,11\}$.

Let $S, T \in \mathcal{F}\left(\mathrm{X}^{2 *}\right)$ where $S=\{\overline{\varepsilon, 0,1}, \overline{00,01,10,11}\}$ and $T=\{\overline{\varepsilon, 0,1}, \overline{00,01,10}, \overline{11}\}$. Then, the following hold:

$S \circledast T=\{\overline{\varepsilon, 0,1}, \overline{00,01,10}, \overline{11}\} \in \mathcal{F}\left(\mathrm{X}^{2 *}\right), \quad S \circledast S=\{\overline{\varepsilon, 0,1}, \overline{00,01,10,11}\}=S$, and $S \circledast T=T \circledast S$. Similarly, results could be computed to show associativity. Thus, $\left(\mathcal{F}\left(\mathrm{X}^{2 *}\right), \circledast\right)$ is a commutative, idempotent monoid with $\{\varepsilon, 0,1,00,01,10,11\}$ as the identity.

$S \oplus T=\{\overline{\varepsilon, 0,1}, \overline{00,01,10,11}\} \in \mathcal{F}\left(\mathrm{X}^{2 *}\right), S \oplus S=\{\overline{\varepsilon, 0,1}, \overline{00,01,10,11}\}=S$, and $S \oplus T=T \oplus S$. Moreover, $I=\{\bar{\varepsilon}, \overline{0}, \overline{1}, \overline{00}, \overline{01}, \overline{10}, \overline{11}\}$ is the identity element since $I \oplus T=\{\bar{\varepsilon}, \overline{0}, \overline{1}, \overline{00}, \overline{01}, \overline{10}, \overline{11}\} \oplus\{\overline{\varepsilon, 0,1}, \overline{00,01,10}, \overline{11}\}=\{\overline{\varepsilon, 0,1}, \overline{00,01,10}, \overline{11}\}=$ $T$, for any $T$. Results could be computed to show that $\oplus$ is associative. Thus, $\left(\mathcal{F}\left(\mathrm{X}^{2 *}\right), \bigoplus\right)$ is a commutative, idempotent monoid with $\{\bar{\varepsilon}, \overline{0}, \overline{1}, \overline{00}, \overline{01}, \overline{10}, \overline{11}\}$ as the identity.

(iii) $S \odot T=\{\overline{\varepsilon, 0,1}, \overline{00,01,10}, \overline{10}\} \in \mathcal{F}\left(\mathrm{X}^{2 *}\right), T \odot S=\{\overline{\varepsilon, 0,1}, \overline{00,01,10,11}\} \in \mathcal{F}\left(\mathrm{X}^{2 *}\right)$, and $T \odot S \neq S \odot T$. Moreover, $T \odot T=\{\overline{\varepsilon, 0,1}, \overline{00,01,10}, \overline{11}\} \odot$ $\{\overline{\varepsilon, 0,1}, \overline{00,01,10}, \overline{11}\}=\{\overline{\varepsilon, 0,1}, \overline{00,01,10}, \overline{11}\}=T$. In order to show associativity, let $R=\{\overline{\varepsilon, 0,1,00}, \overline{01,10}, \overline{11}\} \quad$. Then, $\quad(S \odot T) \odot R=\{\overline{\varepsilon, 0,1}, \overline{00,01,10}, \overline{10}\} \bigcirc$ $\{\overline{\varepsilon, 0,1,00}, \overline{01,10}, \overline{11}\}=\{\overline{\varepsilon, 0,1,00}, \overline{01,10}, \overline{11}\} \quad, \quad$ and $\quad S \odot(T \odot R)=$ $\{\overline{\varepsilon, 0,1}, \overline{00,01,10,11}\} \odot(\{\overline{\varepsilon, 0,1}, \overline{00,01,10}, \overline{11}\} \odot\{\overline{\varepsilon, 0,1,00}, \overline{01,10}, \overline{11}\})=$ $\{\overline{\varepsilon, 0,1}, \overline{00,01,10,11}\} \odot\{\overline{\varepsilon, 0,1,00}, \overline{01,10}, \overline{11}\}=\{\overline{\varepsilon, 0,1,00}, \overline{01,10}, \overline{11}\} \quad$ i.e., $\quad$ (S $\odot$ $T) \odot R=S \odot(T \odot R)$. Thus, $\left(\mathcal{F}\left(\mathrm{X}^{2 *}\right)\right.$, ○) is an idempotent semigroup.

\subsection{Some algebraic structures of a language over $u \in X^{*}$}


Let $u^{*}$ denote the set of all strings over $u \in X^{*}$. Then $u^{*}$ is a commutative monoid under catenation. Moreover, the monoid $C=\left(u^{*}, \circ\right)$ is isomorphic to the monoid $N=(\mathbb{N},$.$) ,$ where $\circ$ and . denote catenation and multiplication, respectively.

\section{Proof}

The first part follows by definition.

For the second part, let $f: C \rightarrow N$ be a function defined as

$$
f(u)=\left\{\begin{array}{l}
1, \text { if } u=\varepsilon, \\
n, \text { if } u=u^{n}, \quad \forall u \in C,
\end{array}\right.
$$

where $u^{n}$ is the $n-$ times catenation of $u$ itself.

It is easy to see that $\forall u, v \in C$, since $f(u v)=f\left(u_{1} u_{2} \ldots u_{n} v_{1} v_{2} \ldots v_{n}\right)=$ $f\left(u_{1}\right) f\left(u_{2}\right) \ldots f\left(u_{n}\right) f\left(v_{1}\right) f\left(v_{2}\right) \ldots f\left(v_{n}\right)=f\left(u_{1} u_{2} \ldots u_{n}\right) f\left(v_{1} v_{2} \ldots v_{n}\right)=f(u) f(v)$, the function $f$ is a monoid homomorphism.

Let $u, v \in C$ such that $f(u)=f(v)$ i.e., $f\left(u_{1}\right) f\left(u_{2}\right) \ldots f\left(u_{n}\right)=f\left(v_{1}\right) f\left(v_{2}\right) \ldots f\left(v_{n}\right)$. Then, as strings are ordered, we have $u_{1}=v_{1}, u_{2}=v_{2}, \ldots, u_{n}=v_{n}$ i.e., $u=v$, which imply that $f$ is injective. Moreover, by the definition of $f, \forall n \in \mathbb{N}, \exists u \in C$ such that $f(u)=n$ i.e., $f$ is surjective.

Hence $f$ is an isomorphism.

\section{Proposition 3.3.1}

A finite $C=\left(u^{*}, \circ\right)$ is a cyclic group of order $n$.

\section{Proof}

Let $u^{*}$ be represented as $\left\{u^{0}, u^{1}, \ldots, u^{n-1}, \ldots\right\}$. A finite $C$ can be represented as $\left(u_{n}^{*}, \circ\right)$ where $u_{n}^{*}$ is the set of $n$ elements of $u^{*}$. Let $C$ be finite viz., $C=\left\{C^{i}, \circ\right\}, i=0,1, \ldots, n-1$, where

$$
C^{i}=\left\{\begin{array}{l}
C^{i+1}, 0 \leq i<n-1 \\
C^{0}, i=n-1 .
\end{array}\right.
$$

Let $C^{i} C^{j}=C^{i+j}, i+j<n$ and $C^{i} C^{j}=C^{i+j-n}, i+j \geq n$. Then, it is easy to see that $C$ is a cyclic group of order $n$.

\section{Example}


Let $\quad u=b b a, u^{*}=\{\varepsilon, b b a, b b a b b a, \ldots\} \quad$ and $n=7 \quad$. Then, $C=$ $\left\{C^{0}, C^{1}, C^{2}, C^{3}, C^{4}, C^{5}, C^{6}\right\}=$

$\{\varepsilon, b b a, b b a b b a, b b a b b a b b a, b b a b b a b b a b b a, b b a b b a b b a b b a b b a, b b a b b a b b a b b a b b a b b a\}$.

Observe that $C^{1} C^{2}=C^{3}, C^{4} C^{5}=C^{2}, C^{6} C^{1}=C^{0}$, etc. Thus, $C$ is a cyclic group of order 7.

\section{Proposition 3.3.2}

Languages of a finite $u^{*}$ form a bounded distributive lattice.

\section{Proof}

Let a finite $u^{*}$ be represented as $u^{n \circledast}=u^{0} \cup u^{1} \cup \ldots \cup u^{n-1}$, and $G$ be the set of all possible languages of $\mathrm{u}^{n \circledast}$. Let $H$ be a structure consisting of $G$ with union and intersection representing the (join) $\vee$ and (meet) $\wedge$ operations, respectively. Let $L_{1}, L_{2}, L_{3} \in G$. It is straightforward to see that $L_{1} \vee L_{2}=L_{2} \vee L_{1}, L_{1} \vee\left(L_{2} \vee L_{3}\right)=\left(L_{1} \vee L_{2}\right) \vee L_{3}$ and $L_{1} \vee$ $L_{1}=L_{1}$ hold, as the union of languages is associative, commutative and idempotent. Thus, $(G, \mathrm{~V})$ is a commutative, idempotent semigroup. Also, as the intersection of languages is commutative, associative and idempotent, $(G, \Lambda)$ is a commutative, idempotent semigroup.

Moreover, as the absorption properties hold i.e., $L_{1} \vee\left(L_{1} \wedge L_{2}\right)=L_{1}$ and $L_{1} \wedge\left(L_{1} \vee L_{2}\right)=L_{1}$, and for all $L_{1}, L_{2} \in G, L_{1} \wedge L_{2}=L_{1}$ and $L_{1} \vee L_{2}=L_{2}$ hold, $H=(G, \vee, \wedge)$ is a lattice.

Also, $\forall L \in G$, as $L \vee \emptyset=L, \emptyset$ is the identity element of the join operation and, as $L \wedge G=L$, $G$ is the identity of the meet operation. Thus, $H$ is a bounded lattice.

In addition, as $L_{1} \vee\left(L_{2} \wedge L_{3}\right)=\left(L_{1} \vee L_{2}\right) \wedge\left(L_{1} \vee L_{3}\right)$ and $L_{1} \wedge\left(L_{2} \vee L_{3}\right)=\left(L_{1} \wedge L_{2}\right) \vee$ $\left(L_{1} \wedge L_{3}\right)$ hold, $H$ is a bounded distributive lattice.

\section{Example}

Let $u=01 \in X^{*}$ over an alphabet $X=\{0,1\}$, and $u^{3 \circledast}=\{\varepsilon, 01,0101\}$. The set $G$ of all possible languages of $\quad u^{3 \circledast}$ is $\{\varnothing,\{\varepsilon\},\{01\},\{0101\},\{\varepsilon, 01\},\{\varepsilon, 0101\},\{01,0101\},\{\varepsilon, 01,0101\}\}$.

Observe that $\{01\} \vee(\{01\} \wedge\{01,0101\})=\{01\}, \quad\{01\} \wedge(\{01\} \vee\{01,0101\})=\{01\}$ i.e., absorption properties hold. Also, $\varnothing \vee\{01,0101\}=\{01,0101\}$ and $\{\varepsilon, 0101\} \wedge$ $\{\varepsilon, 01,0101\}=\{\varepsilon, 0101\}$ i.e., $\emptyset$ is the identity for $\vee$, and $\{\varepsilon, 01,0101\}$ is the identity for $\wedge$. Similarly, results for various other combinations could be computed.

Thus, $(G, \vee, \wedge$,$) is a bounded distributive lattice.$

\section{Concluding Remarks}

A number of operations were introduced on the class of partitions of a language which gave rise to certain monoids and semigroups. Moreover, cyclic group, commutative monoid and 
bounded distributive lattice of a language over a string were introduced. It may be emphasized at this end that the constructions provided in this paper, specially defined on $X^{n *}$, may be found useful to Network segmentation, analysis of large databases, finite state machines, etc. In particular, an alternative representation of a language, developed in definition 2.2, may be exploited for further research.

\section{Acknowledgements}

The authors are thankful to the Editor of The Journal of Mathematics and Computer Science for his suggestion to improve upon the references which has been incorporated.

\section{References}

[1] B. Ahmadi, C. M. Campbell and H. Doostie, "Non-commutative finite monoids of a given order $n \geq 4$ "VERSITA, 22 (2) (2014) 29-35.

[2] J. Gallier, "Introduction to the Theory of Computation", Formal Languages and Automata Models of Computation, Lecture Notes, (2010) 1- 60.

[3] E. Hosseinpour, "T-Rough Fuzzy Subgroups of Groups", The Journal of Mathematics and Computer Science 12 (3) (2014) 186-195.

[4] J. Kari, "Automata and Formal Languages", Lecture Notes, University of Turku, Finland, (2013) $1-150$.

[5] D.E Knuth, "The Art of Computer Programming", Semi-numerical Algorithms, Vol. II, $2^{\text {nd }}$, Addison-Wesley, (1981).

[6] U. Priss, L. J. Old, “Conceptual Structures: Inspiration and Application”, Proceedings of the $14^{\text {th }}$ International Conference on Conceptual Structures ICCS, Denmark, 4068 (2006) 388-400.

[7] J.P. Tremblay, R. Manohar, "Discrete Mathematical Structures with Applications to Computer Science", Tata McGraw-Hill Edition, (1997).

[8] S.A. N. Zadeh, A. Radfar, "A. B. Saied, On BP-algebras and QS-algebras", The Journal of Mathematics and Computer Science, 5 (1) (2012) 17-21. 